\title{
Nanoscale analyses of modified polypropylene microtubes internal surface: an approach covering topographical and force spectroscopic parameters
}

\author{
Eduardo F. Barbosa ${ }^{a, b}$ and Luciano P. Silva ${ }^{a, b *}$
}

A nanoscale characterization of modified and unmodified polypropylene (PP) microtubes internal surface was performed to investigate their structural, chemical, and physical properties. Nanoroughness, stiffness, elasticity, attraction behavior, adhesion forces, and chemical environment were investigated to test some manufacturer statements regarding Axygen MAXYMum Recovery ${ }^{\circledR}$ products. They announced that this class of material presented special features, originated from a modification to the original PP resin and by using a diamond polished mould, providing lower retention and minor interference on laboratorial tests, such as low roughness and little interaction tendency. Then, in this study, modified and control internal surfaces of PP microtubes were compared by atomic force microscopy, scanning electron microscopy, and Fourier transform infrared spectroscopy. Nanoroughness and force spectroscopy parameters assessed by atomic force microscopy showed out as a sensible and high-resolution technique, crucial to discriminate differences between the surfaces. This type of investigation can be considered as a promising approach that can be applied to other polymeric systems, considering nanoscale properties, physical/chemical modifications, and as an alternative route for quality control checking concerning polymeric surfaces. Copyright $\odot 2013$ John Wiley \& Sons, Ltd.

Supporting information can be found in on line version of this article.

Keywords: polypropylene modification; microtube; atomic force microscopy; force spectroscopy; nanoroughness

\section{Introduction}

Polypropylene (PP) devices have been widely applied in worldwide industrial and academic laboratories. ${ }^{[1,2]}$ After microtubes and pipette tips first conception, in the early 80s, their use became ubiquitous in laboratories worldwide. ${ }^{[3]}$ Nevertheless, this material is not as inert as once thought. McDonald et al. (2008) described plasticware, mainly the colorful ones, as a potential source of bioactive contaminants, such as di (2-hydroxyethyl) methyldodecylammonium and 9-octadecenamide (oleamide) ${ }^{[1,2]}$ Then, some manufacturers have been looking for strategies to develop modifications on PP resins and processing steps to overcome these drawbacks: increasing desirable features such as low surface energy and reduced adhesion behavior. Axygen MAXYMum Recovery ${ }^{\circledR}$ microtubes are described by manufacturer as without any potential contaminating coating, by presenting a special polymeric internal surface, which creates low binding features. ${ }^{[4]}$

Chemical structure effects and surface properties of polymeric materials concerning protein adsorption were described as been strongly related to surface roughness. ${ }^{[5]}$ Lower surface roughness means smaller van der Waals contribution to the adhesion force and consequent decreasing of these events. ${ }^{[6]}$ Considering this aspect, Barbosa and Silva investigated PP microtubes internal surface aiming to verify the possible presence of any homogeneity patterns and to relate such information with roughness, adhesion features, and quality control markers. ${ }^{[3,7]}$ They described significant roughness differences both among brands and among the areas along each tube (equal sizes). ${ }^{[7]}$ When comparing tubes with different sizes, the bigger ones presented more heterogeneous surface morphology, which could be related to the adhesion events on polymer surface, since the contact surface area can predicts some reactivity or leaching tendencies considering other materials. ${ }^{[3]}$

The present study aimed to investigate modified microtubes internal surface, compared to unmodified ones. Morphologies, roughness, stiffness, elasticity, attraction, adhesion, and chemical environment were investigated to test the following hypothesis: 'Would PP modified microtubes characterization outcomes corroborate the manufacturer statements about this product (Axygen MAXYMum Recovery $\left.{ }^{\circledR}\right)$ ?' Atomic force microscopy (AFM), scanning electron microscopy (SEM), and Fourier transform infrared spectroscopy (FTIR) were used to assess these properties.

* Correspondence to: Luciano P. Silva, Embrapa Recursos Genéticos e Biotecnologia, Laboratório de Espectrometria de Massa, Prédio da Biotecnologia (PBI), Parque Estação Biológica Final W5 Norte, Brasilia, DF, 70770-917, Brazil

E-mail: luciano.paulino@embrapa.br, lucianopaulinosilva@gmail.com

a Embrapa Recursos Genéticos e Biotecnologia, Laboratório de Espectrometria de Massa, Prédio da Biotecnologia (PBI), Parque Estação Biológica Final W5 Norte, Brasília, DF, 70770-917, Brazil

b Pós-Graduação em Biologia Animal, Instituto de Biologia, Universidade de Brasília, Campus Universitário Darcy Ribeiro, Brasília70910-900, Brazil 


\section{Material and methods}

\section{Sampling}

Two types of commercial PP microtubes ( $0.6 \mathrm{ml}$ of capacity) were selected for topographical and chemical internal surface analyses. Considering the high production reproducibility level and that this methodology presents some limitations related to acquisition time requirements, the analyses of large sample numbers could derail this experiment. Each sample $(n=2)$ was segmented at three different areas along the tube, as illustrated in Fig. 1. Small areas of approximately $6 \mathrm{~mm}^{2}$ were removed from each area to perform the experimental approaches.

\section{Force spectroscopy}

To analyze the surface interaction forces between the tip and microtubes surface, a commercial AFM equipment (SPM-9600, Shimadzu, Japan) was operated in contact mode. A small section of each sample $\left(\sim 6 \mathrm{~mm}^{2}\right)$ was fixed on a metallic support using a double-sided adhesive tape. A pyramidal silicon nitride tip (Olympus, curvature radius $<20 \mathrm{~nm}$ ) was used in this technique. A cantilever with a length of $200 \mu \mathrm{m}$ (spring constant of $0.15 \mathrm{~N} / \mathrm{m}$ and resonance frequency of approximately $24 \mathrm{kHz}$ ) was used. Acquisition rate was at $1 \mathrm{~Hz}$, and the method was loaded at 20 $\mathrm{V}$ of amplitude and $3 \mathrm{~V}$ of operating point. The software used was SPIP v. 5.1.5, set in $23^{\circ} \mathrm{C}$ and $0.15 \mathrm{~N} / \mathrm{m}$ spring constant, cone indentation (Sneddon), five fitting points, and approach curve as base line correction curve. At least 30 force curves analyses were performed to make the reproducibility of the assays safe. All force spectroscopy data were displayed by Origin 8 software and analyzed by one-way ANOVA and Tukey test $(p<0.05)$. Since this mode of measurement did not have any possible standard sample to calibrate the AFM device, the results presented here own value and significance only for comparasion among the samples that we analyzed in this present study.

\section{Nanoroughness characterization}

Samples were analyzed using commercial AFM equipment previously described operating in dynamic phase mode. The scanner used has a $125 \mu \mathrm{m}$ travel in XY-directions and $7 \mu \mathrm{m}$ in the Z-direction. Images were obtained in ambient air, at room temperature $\left(23^{\circ} \mathrm{C}\right)$, and at approximately $20-30 \%$ relative humidity. Scanned areas were perfect squares of $50 \mu \mathrm{m} \times 50 \mu \mathrm{m}$

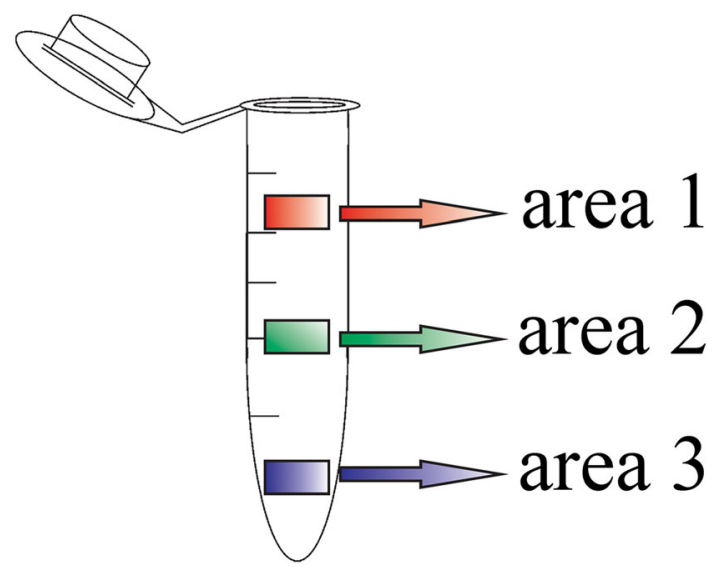

Figure 1. Polypropylene microtube representation of the sectioned areas analyzed by AFM. with $1 \mathrm{~Hz}$ scan rate using an aluminum rectangular cantilever with $125 \mu \mathrm{m}$ length, $320 \mathrm{kHz}$ resonance frequency, $42 \mathrm{~N} / \mathrm{m}$ spring constant, and silicon tip with curvature radius $<10 \mathrm{~nm}$ (Nanosensors PPP-NCHR-20). At least ten areas were scanned of each microtube surface to make the assays reproducibility safe. Considering only one dimension, this sampling represents approximately $12.5 \%$ of the microtube internal length. Trace and retrace procedures were performed in order to prove that samples were not distorted during scanning steps. All AFM images contained $512 \times 512$ data points and were first processed by SPM-9600 off-line software. Processing consisted in an automatic surface X-line, Y-line, plane fit leveling, and two erase noise lines steps. After processing, images surface analysis was performed, and some roughness values were obtained referring to arithmetic mean roughness (Ra), maximum height ( $R z)$, ten point height of roughness profile (Rzjis), root-mean-square roughness $(\mathrm{Rq})$, maximum profile peak height $(\mathrm{Rp})$, and maximum profile valley depth (Rv). Finally, AFM 3D images and surface analyzes (roughness and fractal dimension) were estimated using SPM-9600 off-line software. AFM roughness data were analyzed by Origin 8 software using one-way ANOVA and Tukey test $(p<0.05)$.

\section{Scanning electron microscopy and energy-dispersive X-ray spectroscopy (SEM-EDS)}

Analyses of SEM and EDS were performed in a JSM 700-1F instrument (Jeol, Japan). A small square piece of each sample was fixed on a metallic holders ( $\mathrm{Cu}$ and $\mathrm{Zn}$ ) using a doublesided adhesive tape. Then, they were sputter coated with gold in a Balzers - SCD 050 (Baltec, Austria) at $21^{\circ} \mathrm{C}$, in about $2.6 \times 10^{-7}$ $\mathrm{Pa}$ for $180 \mathrm{~s}$. After coating with gold, samples were analyzed with a voltage of $15 \mathrm{kV}$. EDS analyses were performed after SEM image acquisitions. 'Dead time' parameter values were set to around $20 \%$ by setting the 'current probe' parameter. Image acquisitions were performed under a reduced pressure atmosphere $\left(1.9 \times 10^{-4} \mathrm{~Pa}\right)$.

\section{FTIR}

Infrared absorption spectra were obtained using a Fourier transform infrared spectrometer - 4100 (Jasco, United Kingdom), with wave numbers ranging from 500 to $4000 \mathrm{~cm}^{-1}$. Reported spectra are average of 32 scans. Samples were analyzed in the solid state, where small fragments of each sample were deposited in the attenuated total reflectance system for subsequent acquisition.

\section{Results}

Topographic morphology of unmodified (control) and modified PP microtube internal surface assessed by AFM is presented in Fig. 2. Both groups showed similar surface arrangement with unordered crests crossing the scanned areas. Crests presented discrepancies related to their height. Qualitative observation of the areas along each tube could suggest the existence of substantial differences among them. However, considering the quantitative nanoroughness results, any significant difference was observed $(p<0.05)$.

AFM analyses show some limitations such as maximum analyzed $X-Y$ dimensions and maximum $Z$ axis displacement. To overcome these technical restrictions, SEM analyses were performed to the same surfaces aiming to compare with the qualitative information assessed by AFM. Figure 3 shows control and modified PP microtubes internal surface. SEM results 

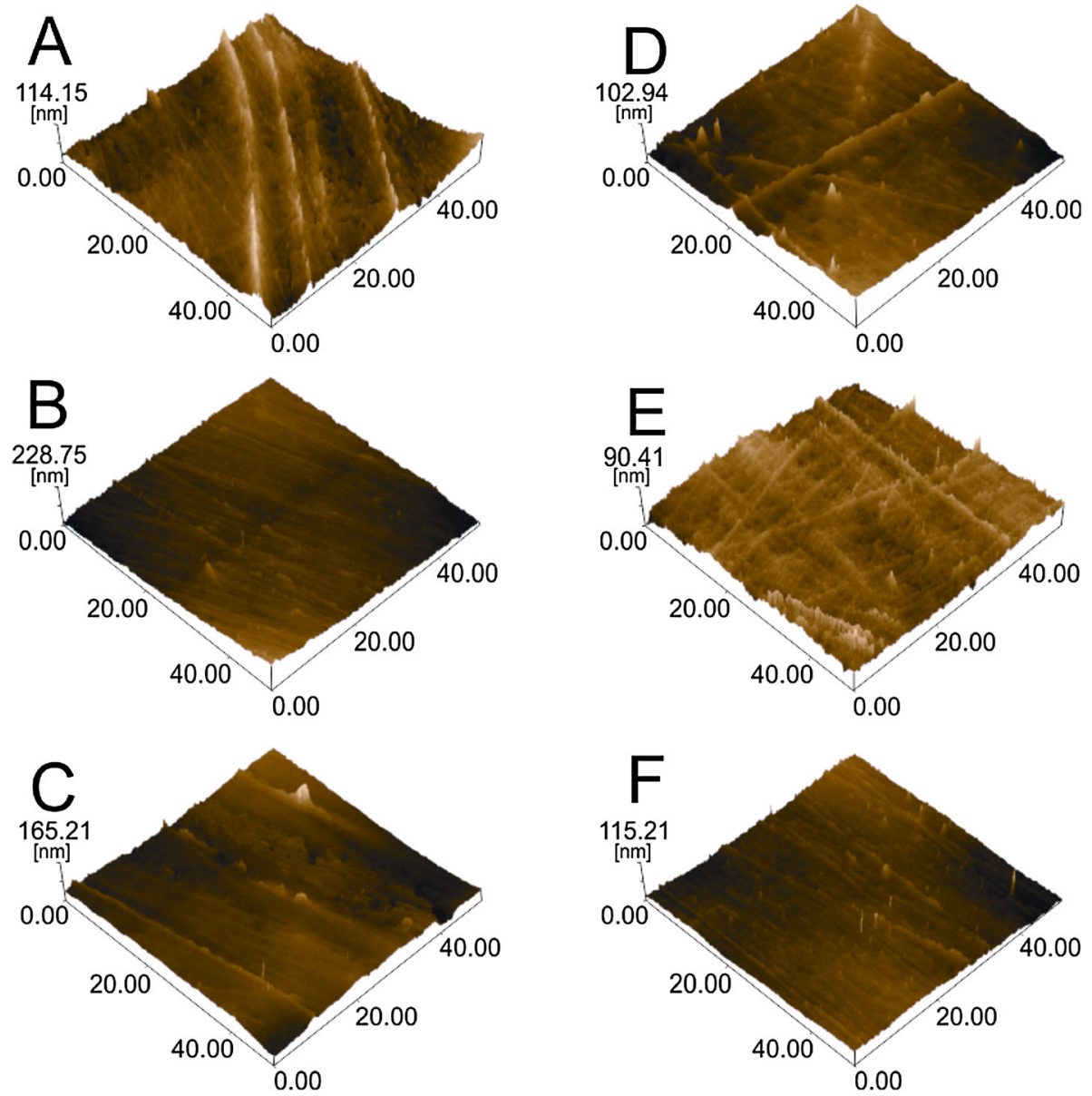

Figure 2. Representative three-dimensional images of PP microtubes obtained by atomic force microscopy (AFM) analyses operating in dynamic mode. Scanning area dimensions ( $x$ and $y$ axis) are represented in micrometer units $(\mu \mathrm{m})$. ' $A, B$, and $C$ ' represent the areas 1,2 , and 3 along each control tube, respectively. ' $\mathrm{D}, \mathrm{E}$, and $\mathrm{F}$ ' represent the areas 1, 2, and 3 along each modified tube, respectively. Microtubes volume was $0.6 \mathrm{ml}$.

A

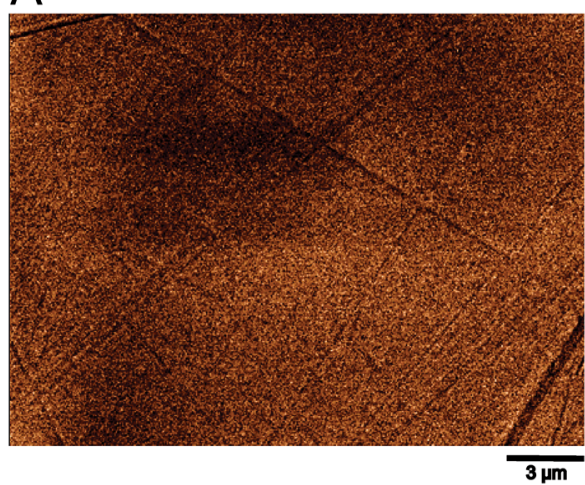

$\mathrm{B}$

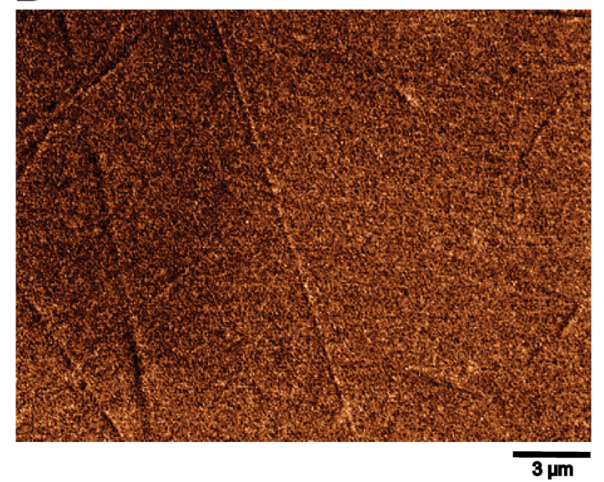

Figure 3. Topographical images obtained by scanning electron microscopy (SEM) of control (A) and modified PP microtube (B). Microtubes volume was $0.6 \mathrm{ml}$.

confirmed the similarity between the surfaces arrangement and allowed the visualization of lines crossing scanned areas, which probably were those observed as crests on AFM analyses.

Since no statistic significant differences were found among each microtube areas, quantitative results presented in Fig. 4 correspond to the arithmetic mean taking into consideration all investigated areas. Nanoroughness outcomes indicated that modified PP microtubes showed reduced $\mathrm{Ra}$ and $\mathrm{Rq}$ roughness results. These parameters represent arithmetic mean and root-mean-square roughness, respectively. Otherwise, modified PP microtubes $\mathrm{Rp}$ roughness results were increased, compared to control $(p<0.05)$.

Figure 5 shows the main force spectroscopy results. Both groups presented significant differences among the areas along each tube. PP modification resulted in a reduction concerning snap in force results and increasing maximum load, maximum pull, and detachment forces. 


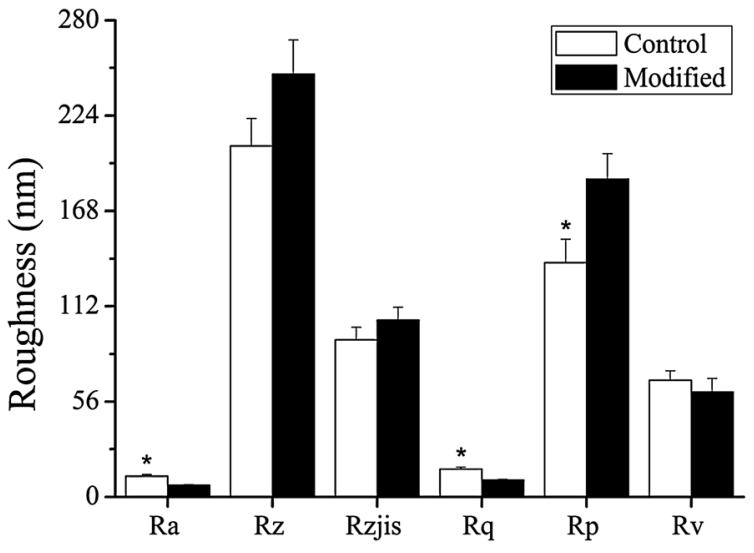

Figure 4. Nanoroughness results obtained by atomic force microscopy (AFM) operating in dynamic mode. Microtubes volume was $0.6 \mathrm{ml}$. Presence of symbol $(*)$ means statistic significant difference between control and modified PP microtubes $(p<0.05)$.

Table 1 shows the elasticity modulus and dissipated energy results estimated from force curve experiments. PP modified microtubes showed Young's Modulus approximately 3.4 times higher than control, while dissipated energy was 1.5 times superior.

Other spectroscopic techniques were used to characterize these surfaces chemical features and aiming to search for some possible features which specify manufacturer described modifications. Figure 6 shows each surface chemical environment obtained by FTIR analysis. Any considerable differences could be detected neither by FTIR results nor by EDS (supplementary material). EDS analyses allow to assess the relative elementary composition of each sample surfaces.

\section{Discussion}

The most relevant structural features observed on both groups are the presence of crests crossing along all analyzed areas (Figs. 2 and 3). According to Thomann et al. (1996), these fibril-like structures are related to the formation of open spherulites in materials containing normal $\alpha$-phase isotactic PP, considering a large-scale image of crystalline entities network structure. ${ }^{[8,9]}$ Quantitative results shown in Fig. 4 related to the peaks roughness highlighted the important contribution of these crest shapes over the modified surface features. Maximum profile peak height roughness (Rp) of modified microtubes was statistically significantly higher than control $(p<0.05)$. Then, increasing peaks contribution on smooth surfaces could conduce to reducing adhesion events, since slighter van der Waals forces participation could take place. ${ }^{[6]}$

Average roughness parameters ( $\mathrm{Ra}$ and $\mathrm{Rq}$ ) indicated $\mathrm{a}$ smoothness increasing on modified PP surfaces (Fig. 4). According to Schönherr et al. (1998), lower average roughness values (Ra) for modified (oxyfluorinated) PP surfaces imply lower total surface free energy. ${ }^{[10]}$ They also described that roughness deviation plays a central hole on force measurements. ${ }^{[10,11]}$

Modified PP microtubes surfaces exhibited unique structural properties (Fig. 5), such as higher stiffness, adhesion, elasticity, and dissipated energy (Table 1). These features are obtained from force curve measurements fitted with the Hertz equation, as used by Griepentrog et al. (2013). ${ }^{[12]}$ Nevertheless, they compared the nanoindentation and AFM methods for mechanical properties determination of polymers aiming to determine the shape and size of AFM tips. ${ }^{[12]}$ In our study, samples surface stiffness behavior was measured by calculating the maximum loading force applied during the indentation step in a force curve assessment. ${ }^{[13]}$ Further than hardness outcomes, this event also allows calculating elasticity or Young's modulus. This property is described as the capacity to return to initial condition or shape after suffering some distortion. ${ }^{[14]}$ Dissipated energy is a work measurement in an approach-retraction cycle and is related to the dynamics of tip trajectory, ${ }^{[15]}$ associating to positional hysteresis and local specific processes. ${ }^{[16]}$ Adhesive properties are calculated as the AFM tip withdraws. Minor adhesive behavior was expected to PP modified surfaces compared to control, since they were flatter. According to Schönherr et al. (1998), significant increased roughness could mask specific interactions between the tip and the sample surface. ${ }^{[10]}$ Then, adhesion results suggest that decreased roughness in modified PP surfaces could provide an enhanced exposition of surface irregularities and an improved interaction between surface and AFM tip atoms. The higher elasticity modulus showed that modified microtubes with reduced amount of occlusions and cavities on their surface presented an improved capacity to return to its initial shape after suffering the distortion caused by AFM tip. Dissipate energy results could suggest that this flattened surface exposes surface irregularities, making possible AFM tip access and providing larger probability of secondary interactions occurrence, increasing energy dissipation processes.
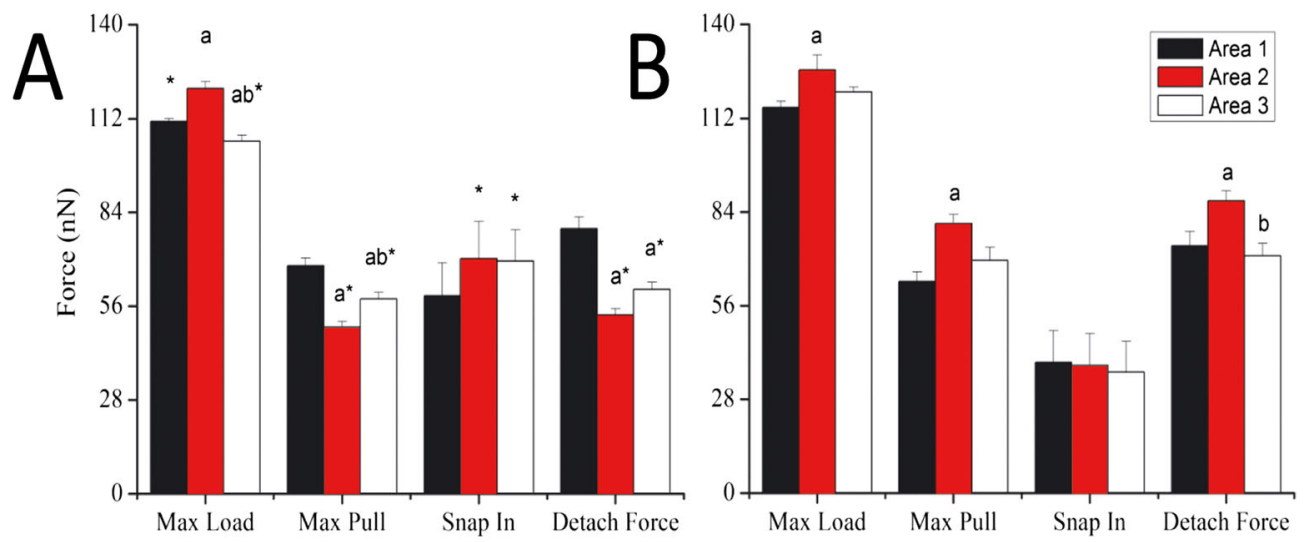

Figure 5. Force spectroscopy results obtained by atomic force microscopy (AFM) for control (A) and modified PP microtubes (B) operating in contact mode. Microtubes volume was $0.6 \mathrm{ml}$. Presence of symbol $\left(^{*}\right)$ means statistically significant difference between control and modified PP microtubes ( $\mathrm{p}$ 0.05 ). Symbol "a" indicates statistically significant difference from part 1 , symbol " $b$ " indicates statistically significant difference from part 2 ( $p<0.05$ ). 
Table 1. Mechanical characterization of control and modified PP microtubes

\begin{tabular}{|lcc|} 
& Young's modulus (MPa) & Dissipated energy $(\mathbf{f} \mathbf{J})$ \\
\hline Control & $7 \pm 2.1$ & $8.5 \pm 0.8$ \\
Modified & $23.8 \pm 7.6$ & $13 \pm 1.1$
\end{tabular}

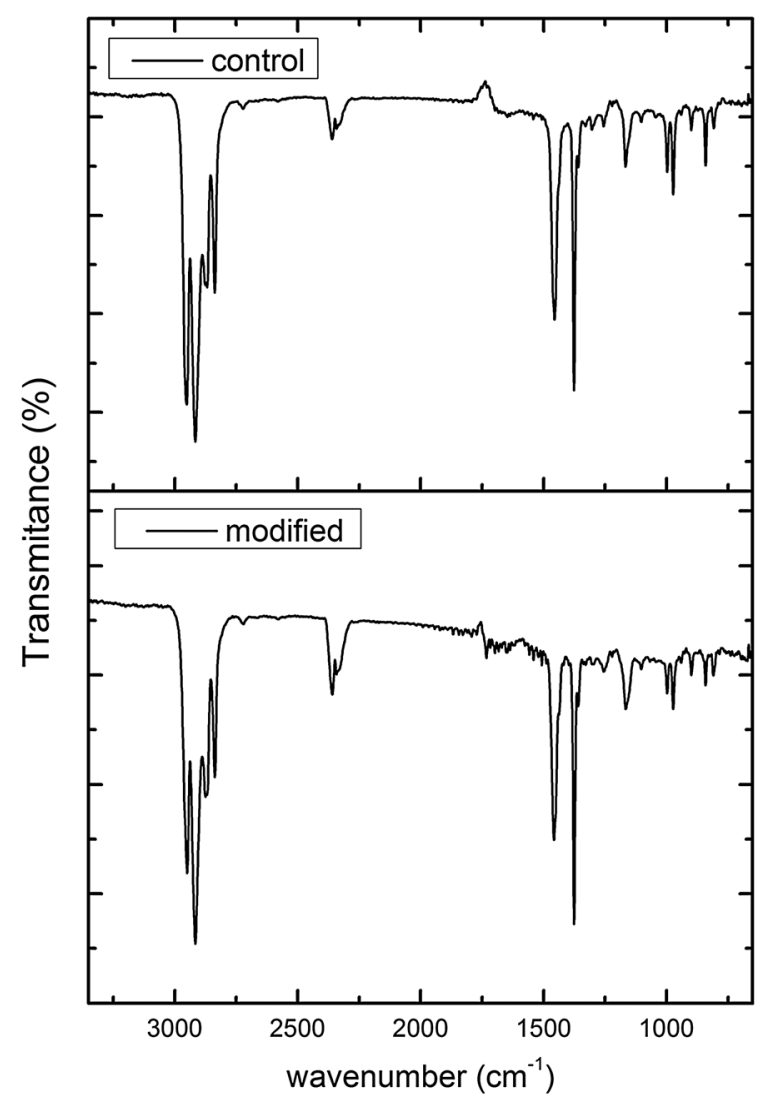

Figure 6. Fourier transform infrared spectroscopy (FTIR) analyses of control (unmodified) and modified PP microtubes internal surface. Measurements were performed with attenuated total reflectance (ATR). Microtubes volume was $0.6 \mathrm{ml}$

Modified PP microtubes showed significant lower attraction force to the AFM tip atoms (Fig. 5). This force is calculated from 'snap in' force parameter and is measured in the beginning of the force curve acquisition (approach step) when the attractive forces outweigh the AFM cantilever spring constant. ${ }^{[13]}$ This property is especially important to corroborate some marketing statements presented by manufacturers, ${ }^{[4]}$ since this outcome suggests a minor interaction tendency relating to this material surface.

These specific properties of modified PP surfaces concerning the prevention of undesirable molecule adhesion events will be assessed in further studies, on which molecules with varied molecular weight, surface charge, and hydrophobicity will be tested considering their adhesion and modifications on polymeric surfaces properties after exposition.

Other topographic property analyzed to these surfaces was the fractal dimension. Fractal dimension of an object is associated to its filling available space capacity. ${ }^{[17]}$ Surface fractal space-filling capacity (fractal dimension or ' $\mathrm{D}$ ') reaches a higher occupancy of its maximally available three-dimensional space than the normal surface. $D=2$ corresponds to a quite smooth surface, while $D=3$ yields a very rough profile. ${ }^{[18]}$ In the present study, both surfaces presented the same values concerning fractal dimension parameter $(D=2)$. Even the control samples, which were rougher than modified ones, did not present fractal properties and can be considered as owing two-dimensional surfaces.

Others spectroscopic techniques did not present resolution to distinguish significant differences between the groups. EDS outcomes revealed similar carbon content for both groups: control $(91.0 \% \pm 5.7)$ and modified microtubes $(93.1 \% \pm 2.7)$. FTIR analyses showed the same chemical environment characteristics as expected for PP crystalline materials (Fig. 6). The frequencies: 805, $840,898,940,972,995,1100$, and $1166 \mathrm{~cm}^{-1}$ are related to $\mathrm{CH}_{2}$ rocking; $\mathrm{C}-\mathrm{C}$ stretching; and $\mathrm{CH}_{3}$ wagging and rocking modes. $1255 \mathrm{~cm}^{-1}$ refers to $\mathrm{CH}_{2}$ twisting and $\mathrm{CH}$ bending; $1455 \mathrm{~cm}^{-1}$ refers to $\mathrm{CH}_{2}$ bending and antisymmetric $\mathrm{CH}_{3}$ bending; $2836 \mathrm{~cm}^{-1}$ refers to symmetric $\mathrm{CH}_{2}$ stretching; 2867 and $2915 \mathrm{~cm}^{-1}$ refers to symmetric $\mathrm{CH}_{3}$ stretching, antisymmetric $\mathrm{CH}_{2}$ stretching, and $\mathrm{CH}$ stretching; and $2960 \mathrm{~cm}^{-1}$ refers to antisymmetric $\mathrm{CH}_{3}$ stretching. ${ }^{[19]}$ This lack of any difference between the tested groups, considering these spectroscopic analyses, strongly suggests that the microtube modification could be related only to the structural processing steps, which directly interfere in the polymerization process and consequently on surface features but not chemical features.

\section{Conclusions}

Modified PP microtubes nanoscale outcomes showed important improvements concerning their internal surface features. Despite the present increase in adhesion strength results compared to control, similar results related to fractal dimension analyses, a combination of lower surface roughness and weaker attractive forces suggests that this kind of material in fact could be more efficient considering the uses in laboratories' daily routine, mainly about sample losses caused by undesirable attachment. Nanoroughness and force spectroscopy parameters determination approached by AFM showed out as a sensible and highresolution technique, essential to discriminate surface features. This type of investigation presented out as a promising approach to evaluate other system features, considering nanoscale properties, physical or chemical modifications, and quality control checking.

\section{Acknowledgments}

The authors acknowledge the financial support from Brazilian agencies MCT/CNPq (processes no. 555.175/2005-7, 484.201/ 2007-7, 302.018/2008-5, and 563.802/2010-3), FAPDF (processes no. $193.000 .445 / 2008$ and $193.000 .429 / 2008$ ), and CAPES (process no. 23038.019088/2009-58). The authors are also grateful to Laboratory of Electron Microscopy and Virology of the Institute of Biology and Laboratory of Environmental Analytical Chemistry of the Institute of Chemistry of the University of Brasilia.

\section{References}

[1] GR McDonald, AL Hudson, SMJ Dunn, H You, GB Baker, RM Whittal, JW Martin, A Jha, DE Edmondson, A Holt, Science 2008, 322, 917.

[2] GR McDonald, J Kozuska, A Holt, G.I.T. Lab. J. 2009, 9, 2-4.

[3] EF Barbosa, LP Silva, in Polypropylene: Synthesis, Applications and Environmental Concerns, NovaScience Pub., New York, 2013, pp. 343-357.

[4] Axygen Catalogue. http: www.biocomdirect.com/pdfs/Axygen_ Catalogue_MCTsect.pdf. Accessed 20 November 2012. 
[5] YX Wang, JL Robertson, WB Spillman, Jr. RO Claus, Pharm. Res. 2004, $21,1362-1373$.

[6] F Podczeck, Particle-particle adhesion in pharmaceutical powder handling, Imperial College, London, 1999, pp 16-34.

[7] EF Barbosa, LP Silva, Surf. Int. Anal. 2012, DOI: 10.1002/sia.3812.

[8] R Thomann, C Wang, J Kressler, R Mülhaupt, Macromolecules 1996 , 29 8425-8434.

[9] GBA Lim, DR Lloyd, Polymer 1993, 33, 513-521.

[10] H Schönherr , Z Hruska, GJ Vancso, Macromolecules, 1998, 31, 3679-3685.

[11] H Schönherr, GJ Vancso, Am. Chem. Soc. Div. Polym. Chem. 1996, 37, 612-613.
[12] M Griepentrog, G Krämer, B Cappella, Polym. Test. 2013, 32, 455-460.

[13] EF Barbosa, LP Silva, J. Memb. Sci. 2012, 407, 128-135.

[14] IN Sneddon, Int. J. Eng. Sci. 1965, 3, 47-57.

[15] M Gauthier, M Tsukada, Phys. Rev. Lett. 2000, 85, 5348-5351.

[16] PM Hoffmann, S Jeffery, JB Pethica, HÖ Özer, A. Phys. Rev. Lett. 2001, $87,265502$.

[17] P Zhang, W Tan, Fresenius J. Anal. Chem. 2001, 369, 302-307.

[18] WG Rothschild, Fractals in chemistry. John Wiley and Sons, New Jersey, 1998.

[19] CY Liang, FG Pearson, J. Mol. Spectrosc. 1960, 5, 290-306. 